\title{
Sensitivity and Specificity of CT and Its signs for Diagnosis of Strangulation in Patients with Acute Small Bowel Obstruction
}

\author{
Ashwini Kumar Jha, ${ }^{1}$ Dr. Wen Hao Tang, ${ }^{2}$ Zhi Bin Bai, ${ }^{2}$ Jia Quan Xiao ${ }^{2}$ \\ 'Department of General Surgery, JMCTH, Janakpurdham, Dhanusha, Nepal, ${ }^{2}$ Department of General Surgery, ZhongDa \\ Hospital Affiliated To SouthEast University, Nanjing, China.
}

\section{ABSTRACT}

Introduction: To perform a meta-analysis to review the sensitivity and specificity of computed tomography and different known computed yomography signs for the diagnosis of strangulation in patients with acute small bowel obstruction.

Methods: A comprehensive Pubmed search was performed for all reports that evaluated the use of CT and discussed different CT criteria for the diagnosis of acute SBO. Articles published in English language from January 1978 to June 2008 were included. Review articles, case reports, pictorial essays and articles without original data were excluded. The bivariate random effect model was used to obtain pooled sensitivity and pooled specificity. Summary receiver operating curve was calculated using Meta-Disc. Software Openbugs 3.0.3 was used to summarize the data.

Results: A total of 12 studies fulfilled the inclusion criteria. The pooled sensitivity and specificity of CT in the diagnosis of strangulation was 0.720 (95\% CI 0.674 to 0.763 ) and 0.866 (95\% CI 0.837 to 0.892) respectively. Among different CT signs, mesenteric edema had highest Pooled sensitivity of 0.741 and lack of bowel wall enhancement had highest pooled specificity of 0.991 .

Conclusions: This review demonstrates that CT is highly sensitive as well as specific in the preoperative diagnosis of strangulation SBO which are in accordance with the published studies. Our analysis also shows that "presence of mesenteric fluid" is most sensitive, and "lack of bowel wall enhancement" is most specific CT sign of strangulation, and also justifies need of large scale prospective studies to validate the results obtained as well as to determine a clinical protocol.

Keywords: Acute small bowel obstruction; bowel Ischemia; Computed Tomography; strangulation.

\section{INTRODUCTION}

In the management of Small bowel obstruction(SBO), patients with ischemia or irreversible complete obstruction needs to be differentiated from those with reversible and non-life threatening conditions. ${ }^{1}$ Reported prevalence of strangulating SBO ranges from $5 \%-42 \%,{ }^{2,3}$ requiring urgent intervention to prevent bowel necrosis, sepsis, and death. ${ }^{4-6} \mathrm{~A}$ strangulating obstruction has a mortality rate of $20 \%$ to $37 \% .^{7-10}$

Clinical and laboratory findings as well as radiological tools except CT are of limited value in diagnosing strangulating SBO. ${ }^{11-13}$ Recent works suggested that CT is a reliable modality for identification of ischemic bowel, with sensitivity $>90 \%$ and specificity about $100 \% .{ }^{14-}$ 18 Using CT criteria reported in most literatures, ${ }^{19-28}$ this concondrum of strangulating obstruction is often

Correspondence: Dr. Ashwini Kumar Jha, Department of Surgery, Janaki Medical College, Janakpur, Nepal. E-mail: drashwinikumariha@gmail.com, Phone: +977-9842494827. 
Jha et al. Sensitivity and Specificity of CT and Its signs for Diagnosis of Strangulation in Patients with Acute Small Bowel...

solved. Due to the pathophysiological changes in obstruction and accuracy of the interpreters, some CT indicators previously described as highly specific for strangulating SBO are also seen in simple obstruction. Thus, this study was conducted to review sensitivity and specificity of CT and its signs for preoperative diagnosis of strangulation in patients with acute SBO.

\section{METHODS}

Literature Search: A comprehensive literature search for English language articles was performed using the PubMed database, from Jan 1978 to June 2008. The key words were: ("Small bowel obstruction" [ MeSH ] ) AND ( "Intestinal obstruction" [ $\mathrm{MeSH}]$ ) AND ( "Strangulating bowel obstruction" [MeSH ] ) AND ( "Tomography, x-ray computed" [MeSH ] ) AND ( sensitivity and specificity [ MeSH ] ) OR sensitivity [ WORD ] ) OR specificity [ WORD ] ) OR false positive [ WORD ] OR false negative [ WORD ] OR diagnosis [ MeSH ] OR diagnostic use [ MeSH ] OR detection [ WORD].

Other databases, such as EMBASE and Cochrane controlled trial register, were also checked for relevant articles with the following key words: Small bowel obstruction [ $\mathrm{MeSH}]$ ) AND Intestinal obstruction [ $\mathrm{MeSH}$ ] ) AND Strangulating bowel obstruction [MeSH ] ) AND Tomography, x-ray computed [MeSH ] ). Review articles, letters, comments, case reports, abstracts only, pictorial essays and articles without original data were not selected. The lists of articles were supplemented with extensive cross - checking of the reference lists of all retrieved articles.

Selection of Studies: Four observers independently checked all retrieved articles for inclusion criteria, according to established methodological standards for evaluation of diagnostic tests. ${ }^{29,30}$ One observer (A.K.J.) checked all articles and withdrawn the datas. Any confusion was resolved by consulting other three observers (W.H.T., Z.B.B., and J.Q.X.). The inclusion criteria were as follows: (a) Articles had to be written in English, include primary data, and be published in a peer review journal. (b) Studies should be performed on human subjects with disease of interest. (c) Studies mentioning CT and different CT signs of ischemia in cases of acute small bowel obstruction. (d) Studies in which surgical diagnosis, pathological diagnosis or autopsy reports was used for reference standard. The exclusion criteria were as follows: (a) Studies done on animal models. (b) Articles published in other languages than English. (c) Article with very small sample size $<10$ were excluded. (d) Studies performed to focus on the role of $\mathrm{CT}$ but not explaining the $\mathrm{CT}$ findings of ischemia in cases of acute SBO (e) Studies performed to focus individual CT signs of ischemia in cases of acute SBO. (f) Articles published from the same departments of an institution.

Data Extraction: One observer (A.K.J.) extracted data from all articles by using a standardized form. The QUADAS quality assessment tool was used to extract relevant study design characteristics of each study. ${ }^{31}$ Any problem during data extraction was resolved by consultation with other three observers (W.H.T, Z.B.B. and J.Q.X.). The data from each article was extracted being aware of the site of origin of publication, journal and year of publication. The following data were recorded for each article: (a) author and year of publication, (b) type of study, (c) sample size, (d) diagnosis of strangulation by CT among the total sample size, (e) the sensitivity and specificity of diagnosis of strangulation by $\mathrm{CT}$, (f) the number of patients having different CT signs of strangulation like bowel wall thickening, ascites, lack of bowel wall enhancement, mesenteric edema, mesenteric haziness, mesenteric vascular engorgement, target configuration of bowel wall. For different CT signs of small bowel strangulation, following definitions were considered as reference standards: (a) Bowel wall thickening: Because of difficulties related to the precision of this measurement, we have decided to include the studies which used bowel wall thickening of $\geq 2.5 \mathrm{~mm}$ as an standard. (b) Ascites: Defined as the presence of fluid in the peritoneal cavity as opposed to fluid in the mesentery. (c) Lack of bowel wall enhancement: No contrast enhancement of the bowel wall was considered to be present when there was no enhancement of bowel wall compared with that of the adjacent wall. (d) Mesenteric fluid: The presence of localized fluid in the small bowel mesentery attached to the abnormal (dilated, thickened) small bowel loops was considered abnormal. (e) Mesenteric haziness: Enlargement of the small mesenteric veins around the site of the obstruction as compared to with mesenteric veins distant from this site was considered a positive finding. (f) Engorgement of mesenteric vessels: The mesenteric vessels were engorged when they exceeded $3 \mathrm{~mm}$ in diameter or where there was a localized increase in the number of vessels. (g) Target configuration of bowel wall: Defined as the presence of transition zone with dilation of bowel proximally, decompression of bowel distally, intraluminal contrast that does not pass beyond the transition zone, and a colon containing little gas or fluid. (h) Serrated beak sign: Saw - toothed appearance at the tapering ends of the closed loops. (i) Smooth beak sign: Characterized by presence of fusiform tapering at the site of obstruction in the longitudinal section.

Data Analysis: Different aggregated data sets were 
Jha et al. Sensitivity and Specificity of CT and Its signs for Diagnosis of Strangulation in Patients with Acute Small Bowel...

constructed - the first including studies that reported the CT diagnosis of bowel strangulation in patients with acute SBO, and the others including studies that reported the known $\mathrm{CT}$ signs of strangulation in patients with acute SBO. Since our analysis included the studies explaining both $\mathrm{CT}$ and $\mathrm{CT}$ signs together as a single paper, few studies are repeated in the data sets. All the possible informations from the included articles were retrieved. Studies conducted by Kim et al and Sheddy et al. ${ }^{14,18}$ were used for more than one time in order to extract more information. After extraction of data, pooled sensitivity and specificity, predicted sensitivity and specificity, and weighted values were calculated using Bayesian bivariate random effect model. The greater the values, the more significant the results. Relative credibility was calculated to compare the strength of evidence provided by retrospective and prospective studies. The values greater than one shows that the results provided by the retrospective have more significance in comparison to the results provided by the prospective studies. Summary receiver operating curve was calculated using Meta-Disc. Density graphs were constructed using software Openbugs 3.0.3.

\section{RESULTS}

Study description: There were 12 studies included in the analysis (Table 1). Most of the studies described the patient population or recruitment procedure in detail. All studies used surgery as the reference standard for detection of strangulation. All 12 studies described the scanner model, scanning method, defined clinical criteria for disease confirmation, and defined the criteria for test interpretation.

\begin{tabular}{|c|c|c|c|c|c|c|c|c|}
\hline Author & Journal & Year & Type of study & $\begin{array}{l}\text { Sample } \\
\text { size }\end{array}$ & $\begin{array}{l}\text { Diagnosis of } \\
\text { strangulation } \\
\text { by Surgery }\end{array}$ & $\begin{array}{c}\text { Diagnosis of } \\
\text { strangulation by } \mathrm{CT}\end{array}$ & $\begin{array}{l}\text { Sensitivity and } \\
\text { specificity of } \\
\text { CT mentioned }\end{array}$ & $\begin{array}{c}\text { CT signs of } \\
\text { strangulation } \\
\text { present }\end{array}$ \\
\hline $\begin{array}{c}\text { Kim et } \\
\mathrm{al}^{\left[{ }^{[14]}\right.}\end{array}$ & World J surg & 2004 & Retrospective & 136 & 66 & 66 & Yes & yes \\
\hline Ha et al ${ }^{[15]}$ & Radiology & 1997 & Retrospective & 84 & 41 & 41 & Yes & yes \\
\hline $\begin{array}{l}\text { Donckier } \\
\text { et al[16] }\end{array}$ & $\begin{array}{l}\text { British Journal } \\
\text { of Surgery }\end{array}$ & 1998 & Prospective & 54 & 16 & 16 & Yes & yes \\
\hline $\begin{array}{l}\text { Zalcman } \\
\text { et al[17] }\end{array}$ & AJR & 2000 & Prospective & 144 & 24 & 24 & Yes & yes \\
\hline $\begin{array}{c}\text { Sheedy et } \\
\qquad \mathrm{al}^{[18]}\end{array}$ & Radiology & 2006 & Prospective & 61 & 27 & 27 & Yes & yes \\
\hline $\begin{array}{l}\text { Balthazar } \\
\text { et al[19] }\end{array}$ & Radiology & 1997 & Prospective & 100 & 24 & 24 & Yes & yes \\
\hline $\begin{array}{l}\text { Balthazar } \\
\text { et al[20] }\end{array}$ & Radiology & 1992 & Retrospective & 19 & 16 & 16 & Yes & yes \\
\hline $\begin{array}{c}\text { Frager et } \\
\mathrm{al}^{[23]}\end{array}$ & AJR & 1996 & Prospective & 60 & 29 & 29 & Yes & yes \\
\hline Ha et al ${ }^{[24]}$ & $\begin{array}{c}\text { J Comput } \\
\text { Assist Tomogr }\end{array}$ & 1993 & Retrospective & 20 & 10 & 10 & No & yes \\
\hline $\begin{array}{l}\text { Yen et } \\
\text { al[32] }\end{array}$ & $\begin{array}{l}\text { J Chin Med } \\
\text { Assoc }\end{array}$ & 2005 & Retrospective & 78 & 16 & 16 & No & yes \\
\hline $\begin{array}{c}\text { Catel et } \\
\text { al[ }^{[33]}\end{array}$ & J Radiol & 2003 & Retrospective & 43 & 15 & 14 & No & yes \\
\hline $\begin{array}{c}\text { Taourel et } \\
\mathrm{al}^{[34]}\end{array}$ & AJR & 1995 & Prospective & 52 & 12 & 9 & Yes & Yes \\
\hline
\end{tabular}

In study conducted by Kim et al. ${ }^{14}$ the sensitivity and specificity of CT in the diagnosis of strangulation in acute SBO was interpreted by three different readers blinded to the clinical or other findings of the patient. In our analysis, we included a pooled data from the three different readers to evaluate the sensitivity and specificity of CT in the diagnosis of strangulation in patients with acute SBO. Similarly, article published by Kim et al in 2004 has analyzed the radiological findings by dividing the patients in true and false interpretation groups (taking surgical finding as a standard of reference) for the evaluation of CT signs of strangulation in patients with acute SBO. We used data from both the groups in our analysis for more accuracy. 
In study conducted by Sheddy et al. ${ }^{18}$ it was possible to extract more information as it conducted both prospective and retrospective analysis and presented the datas separately for sensitivity and specificity of CT in the diagnosis of strangulation in patients with acute small bowel obstruction.

A) Results of analysis for the role of CT to diagnose strangulation:
The sensitivity, specificity, positive likelihood ratios and negative likelihood ratios were calculated for each study (Table 2). The presence of different CT signs indicated that strangulation can be diagnosed pre-operatively by CT with a sensitivity ranging from $14.8 \%$ to $100 \%$, specificity $61 \%$ to $100 \%$, positive likelihood ratio 2.517 to 15.042 and negative likelihood ratio 0.027 to 0.905 . The pooled sensitivity and specificity of CT for bowel ischemia in acute SBO were $72 \%$ and $86 \%$ respectively, with positive and negative likelihood ratios of 5.844 and 0.263 respectively (Table 2 ).

\begin{tabular}{|c|c|c|c|c|c|c|c|c|c|c|c|c|}
\hline Reference & Year & Type & Total & TP & FP & TN & FN & $\begin{array}{c}\text { Sensi- } \\
\text { tivity } \\
(\%)\end{array}$ & $\begin{array}{c}\text { Speci- } \\
\text { ficity } \\
(\%)\end{array}$ & $\mathbf{L R}+$ & LR- & DOR \\
\hline $\begin{array}{l}\text { Kim et } \\
\mathrm{al}^{[14]}\end{array}$ & 2004 & Retrospective & 408 & 139 & 40 & 59 & 170 & 70.2 & 81 & 3.686 & 0.368 & 10.013 \\
\hline Ha et $\mathrm{al}^{[15]}$ & 1997 & Retrospective & 84 & 35 & 6 & 6 & 37 & 85.4 & 86 & 6.118 & 0.170 & 35.972 \\
\hline $\begin{array}{l}\text { Donckier } \\
\text { et } \mathrm{al}^{[16]}\end{array}$ & 1998 & Prospective & 54 & 16 & 3 & 0 & 35 & 100 & 92.1 & 10.815 & 0.032 & 334.71 \\
\hline $\begin{array}{l}\text { Zalcman } \\
\text { et al }{ }^{[17]}\end{array}$ & 2000 & Prospective & 144 & 23 & 9 & 1 & 111 & 95.8 & 92.5 & 12.778 & 0.045 & 283.67 \\
\hline $\begin{array}{l}\text { Sheddy et } \\
\mathrm{al}^{[18]}\end{array}$ & 2006 & Prospective & 61 & 4 & 2 & 23 & 32 & 14.8 & 94.1 & 2.519 & 0.905 & 2.783 \\
\hline $\begin{array}{l}\text { Sheddy et } \\
\mathrm{al}^{[18]}\end{array}$ & 2006 & Retrospective & 61 & 14 & 4 & 13 & 30 & 51.9 & 88.2 & 4.407 & 0.546 & 8.077 \\
\hline $\begin{array}{l}\text { Balthazar } \\
\text { et } \mathrm{al}^{[19]}\end{array}$ & 1997 & Prospective & 100 & 19 & 4 & 5 & 72 & 79.2 & 94.7 & 15.042 & 0.220 & 68.400 \\
\hline $\begin{array}{l}\text { Balthazar } \\
\text { et } \text { al }^{[20]}\end{array}$ & 1992 & Retrospective & 19 & 10 & 0 & 6 & 3 & 62.5 & 100 & 4.941 & 0.437 & 11.308 \\
\hline $\begin{array}{l}\text { Frager et } \\
\mathrm{al}^{[23]}\end{array}$ & 1996 & Prospective & 60 & 29 & 12 & 0 & 19 & 100 & 61.3 & 2.517 & 0.027 & 92.040 \\
\hline $\begin{array}{l}\text { Taourel et } \\
\mathrm{al}^{34]}\end{array}$ & 1995 & Prospective & 52 & 9 & 4 & 3 & 36 & 75 & 90 & 7.500 & 0.278 & 27.000 \\
\hline Pooled & & & 1043 & 298 & 84 & 116 & 545 & 72 & 86.6 & 5.844 & 0.263 & 25.981 \\
\hline
\end{tabular}

A summary ROC curve was constructed from the pooled data, using both non-weighted and weighted analyses; the

area under curve was 0.98 (Fig 1).

The timing of abdominal CT and the abdominal exploration differed among the studies, ranging from few hours to 16 days. The diagnostic odds ratio (DOR) ranges from 2.783 to 334.71 , with a pooled DOR of 25.981 (95\% Cl $11.176-60.399)$.
The calculation of relative credibility (RC) showed that the evidence provided by retrospective studies is stronger than those of prospective studies, for sensitivity and specificity of CT in the diagnosis of strangulation in patients with acute SBO.

B) Results of analysis for evaluation of the role of different CT signs of strangulation (Table 3): 


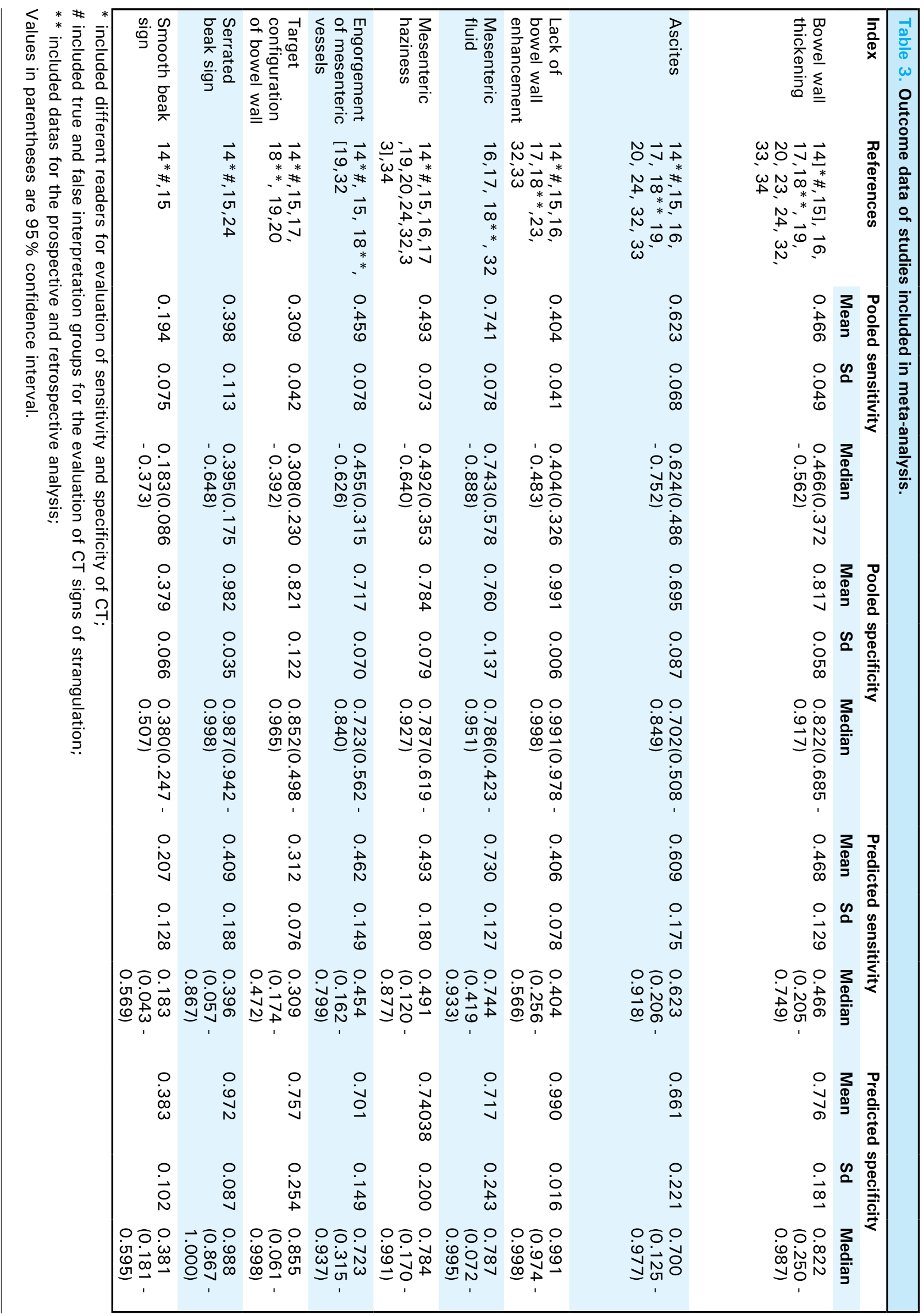


Jha et al. Sensitivity and Specificity of CT and Its signs for Diagnosis of Strangulation in Patients with Acute Small Bowel...

a) Bowel wall thickening: Seven hundred and ninety three patients were evaluated for the presence of this CT sign in all of the included studies. ${ }^{14-20,23,24,32-34}$ The reported sensitivity and specificity in the included studies ranges from $21 \%$ to $100 \%$ and $25 \%$ to $100 \%$ respectively. The pooled sensitivity and specificity are $0.466(95 \% \mathrm{Cl} 0.372,0.562)$ and $0.822(95 \% \mathrm{Cl}$ $0.685,0.917)$ respectively, with a predicted sensitivity and specificity of $0.466(95 \% \mathrm{Cl} 0.205,0.749)$ and 0.822 (95\% Cl 0.250, 0.987) respectively.

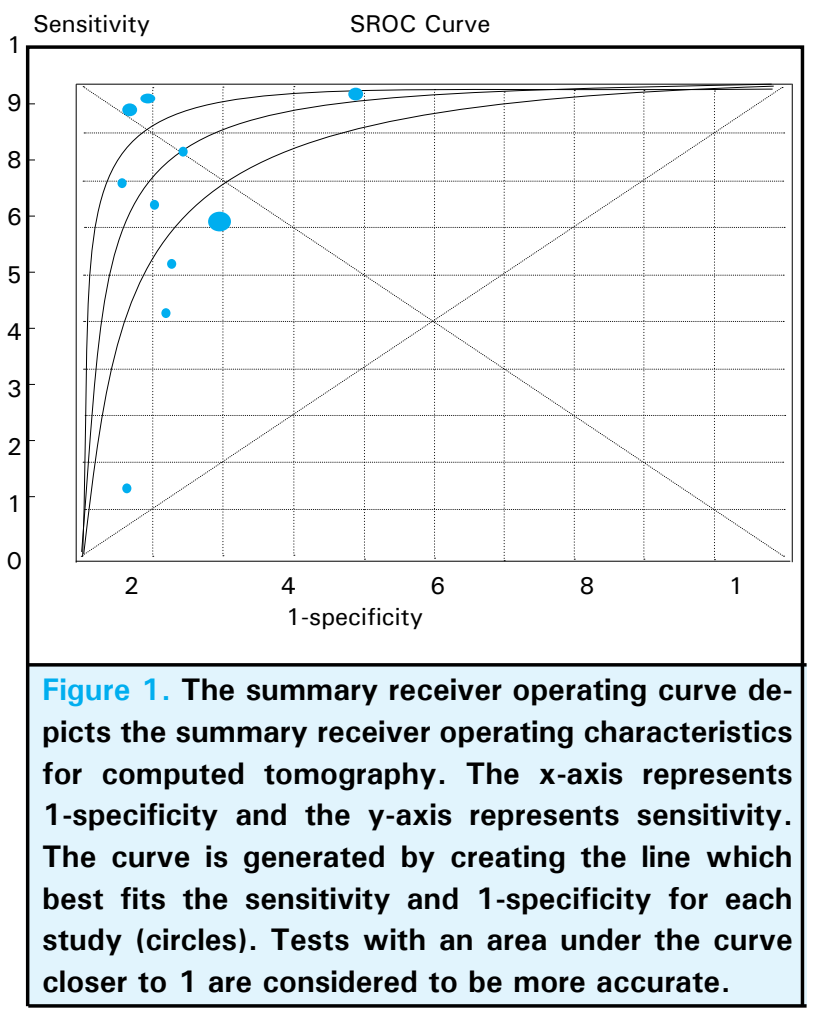

b) Ascites: The sensitivity and specificity of ascites for the diagnosis of strangulation in patients with acute SBO was evaluated in ten studies. ${ }^{14-20,24,32,33}$ The total numbers of patients evaluated for the presence of ascites were Seven hundred sixteen. The included studies had reported sensitivity and specificity ranging from $16 \%$ to $90 \%$ and $37 \%$ to $98 \%$ respectively. In our analysis, the pooled sensitivity and specificity are $0.624(95 \% \mathrm{Cl} 0.486,0.752)$ and $0.702(95 \% \mathrm{Cl}$ $0.508,0.849$ ) respectively, with a predicted sensitivity and specificity of $0.623(95 \% \mathrm{Cl} 0.206,0.918)$ and $0.700(95 \% \mathrm{Cl} 0.125,0.977)$ respectively.

c) Lack of bowel wall enhancement: Eight studies, ${ }^{14-18,23,32,33}$ with a sample size of six hundred and forty nine, have focused on the effect of presence or absence of this $\mathrm{CT}$ sign on the overall diagnosis of strangulation in the setting of acute SBO. The reported sensitivity and specificity in the included studies ranges from $21 \%$ to $59 \%$ and $92 \%$ to $100 \%$ respectively.
The pooled sensitivity and specificity are $0.404(95 \%$ $\mathrm{Cl} 0.326,0.483)$ and $0.991(95 \% \mathrm{Cl} 0.978,0.998)$ respectively, with a predicted sensitivity and specificity of $0.404(95 \% \mathrm{Cl} 0.256,0.566)$ and $0.991(95 \% \mathrm{Cl}$ $0.974,0.998)$ respectively.

d) Mesenteric fluid: Two prospective studies ${ }^{16,17}$ with one hundred ninety eight patients and two retrospective studies $^{18,32}$ with one hundred thirty nine patients, have analyzed the sensitivity and specificity of this CT sign for the diagnosis of strangulation in case of acute SBO. Its sensitivity and specificity in the included studies varies from $52 \%$ to $94 \%$ and $41 \%$ to $100 \%$ respectively. The pooled sensitivity and specificity are $0.743(95 \%$ $\mathrm{Cl} 0.578,0.888)$ and $0.786(95 \% \mathrm{Cl} 0.423,0.951)$ respectively, with a predicted sensitivity and specificity of $0.744(95 \% \mathrm{Cl} 0.419,0.933)$ and $0.787(95 \%$ $\mathrm{Cl} 0.072,0.995)$ respectively. The calculation of relative credibility showed that the data presented in retrospective studies are more supportive to this CT sign than that of from the prospective studies.

e) Mesenteric haziness: The reported sensitivity and specificity in ten studies ${ }^{14-17,19,20,24,32,33,34}$ varies from $5 \%$ to $100 \%$ and $48 \%$ to $100 \%$ respectively. A total of six hundred and seventy two patients were evaluated for presence of this CT sign. The pooled sensitivity and specificity are $0.743(95 \% \mathrm{Cl} 0.578,0.888)$ and $0.787(95 \% \mathrm{Cl} 0.619,0.927)$ respectively, with a predicted sensitivity and specificity of 0.491 (95\% $\mathrm{Cl} 0.120,0.877)$ and $0.784(95 \% \mathrm{Cl} 0.170,0.991)$ respectively. The comparison between studies shows a poor evidence of data provided by prospective study for the evaluation of this CT sign.

f) Engorgement of mesenteric vessels: A total of five studies $^{14,15,18,19,32}$ have reported the sensitivity (19\% to $71 \%)$ and specificity (42\% to $92 \%$ ) of this CT sign. The pooled sensitivity and specificity are $0.455(95 \%$ $\mathrm{Cl} 0.315,0.626)$ and $0.723(95 \% \mathrm{Cl} 0.562,0.840)$ respectively, with a predicted sensitivity and specificity of $0.454(95 \% \mathrm{Cl} 0.162,0.799)$ and $0.723(95 \% \mathrm{Cl}$ $0.315,0.937)$ respectively.

g) Target configuration of bowel wall: There were six studies $^{14,15,17-20}$ which reported the data in terms of sensitivity ranging from $19 \%$ to $41 \%$ and specificity ranging from $41 \%$ to $99 \%$. The pooled sensitivity and specificity are $0.308(95 \% \mathrm{Cl} 0.230,0.392)$ and 0.852 (95\% Cl 0.498, 0.965) respectively, with a predicted sensitivity and specificity of $0.309(95 \% \mathrm{Cl} 0.174$, $0.472)$ and $0.855(95 \% \mathrm{Cl} 0.061,0.998)$ respectively.

h) Serrated beak sign: Only three studies $^{14,15,24}$ had included two hundred and forty patients for the evaluation of this CT sign of strangulation, and reported the sensitivity of $15 \%$ to $47 \%$ and specificity of $92 \%$ 
Jha et al. Sensitivity and Specificity of CT and Its signs for Diagnosis of Strangulation in Patients with Acute Small Bowel...

to $100 \%$. The pooled sensitivity and specificity are $0.395(95 \% \mathrm{Cl} 0.175,0.648)$ and $0.987(95 \% \mathrm{Cl}$ $0.942,0.998)$ respectively, with a predicted sensitivity and specificity of $0.396(95 \% \mathrm{Cl} 0.057,0.867)$ and $0.988(95 \% \mathrm{Cl} 0.867,1.000)$ respectively.

i) Smooth beak sign: The sensitivity ranging from $11 \%$ to $47 \%$ and specificity ranging from $25 \%$ to $42 \%$ of this CT sign can be extracted only from two studies. ${ }^{14,15}$ These two studies had evaluated the CT findings of three hundred twenty patients. The pooled sensitivity and specificity are $0.183(95 \% \mathrm{Cl} 0.086,0.373)$ and $0.380(95 \% \mathrm{Cl} 0.247,0.507)$ respectively, with a predicted sensitivity and specificity of $0.183(95 \%$ $\mathrm{Cl} 0.043,0.569)$ and 0.381 (95\% Cl 0.181, 0.595) respectively.
C) Comparison of prospective and retrospective studies:

The overall comparison of the data reported by the prospective and retrospective studies enrolled in our analysis for every separate index were done, and values were presented in terms of relative credibility $(\mathrm{RC})$. The values greater than one indicate that the evidence of data provided by the prospective studies were poorer than that of retrospective studies (Table 4). The relative credibility of CT is $1.664(95 \% \mathrm{Cl} 0.563,4.429)$. In case of CT sign, the data collected for every signs shows that the results provided by retrospective studies are more stronger than those of prospective studies, with target configuration of bowel wall being the highest, with a mean relative credibility value of 11.757 (95\% $\mathrm{Cl} 2.183,49.181)$. These results indicate the need of performance of large scale prospective studies.

\begin{tabular}{|c|c|c|c|c|}
\hline \multirow[t]{2}{*}{ Index } & \multirow[t]{2}{*}{ References } & \multicolumn{3}{|c|}{ Relative credibility } \\
\hline & & Mean & Sd & Median \\
\hline CT & $\begin{array}{l}14 * \#, 15,16,17,18 * *, 19,20,23 \\
34\end{array}$ & 1.664 & 1.100 & $1.384(0.563-4.429)$ \\
\hline Bowel wall thickening & $\begin{array}{l}14 * \#, 15,16,17,18 * *, 19,20 \\
23,24,32,33,34\end{array}$ & 2.337 & 1.056 & $2.152(0.975-4.947)$ \\
\hline Ascites & $\begin{array}{l}14 * \#, 15,16,17,18 * *, 19,20,24 \\
32,33\end{array}$ & 4.355 & 2.842 & $\begin{array}{l}3.823(1.405- \\
10.806)\end{array}$ \\
\hline $\begin{array}{l}\text { Lack of bowel wall } \\
\text { enhancement }\end{array}$ & $14^{*} \#, 15,16,17,18^{* *}, 23,32,33$ & 2.281 & 1.193 & $2.039(0.945-5.120)$ \\
\hline Mesenteric fluid & $16,17,18 * *, 32$ & 2.042 & 4.797 & $\begin{array}{l}1.048(0.110- \\
10.230)\end{array}$ \\
\hline Mesenteric haziness & $\begin{array}{l}\left.14^{*} \#, 15,16,17,19,20,24\right], 32 \\
33,34\end{array}$ & 2.122 & 1.173 & $1.902(0.682-4.962)$ \\
\hline $\begin{array}{l}\text { Target configuration of } \\
\text { bowel wall }\end{array}$ & $14^{*} \#, 15,17,18^{* *}, 19,[20$ & 11.757 & 28.758 & $6.931(2.183-49.181)$ \\
\hline
\end{tabular}

studies have poorer evidence than retrospective studies.

\section{DISCUSSION}

In this systemic review, we found that CT is a highly accurate modality in the diagnosis of strangulation in patients with acute small bowel obstruction with the highest pooled sensitivity and specificity of $72 \%$ and $86.6 \%$ respectively. Early detection of bowel ischemia in acute SBO is critical because it mandates operation, ${ }^{35-39}$ as ischemia is associated with an increased mortality rate. ${ }^{40}$ Because ischemia is unlikely to resolve without surgical intervention, ${ }^{25,41,42}$ resolution without surgery suggests that ischemia was not present and therefore CT scan appears to be an important modality in detecting ischemia. ${ }^{43,44}$ This review also evaluated the well-defined signs of ischemia that can be seen on CT, like bowel wall thickening, ascites, mesenteric haziness, mesenteric vessel engorgement, lack of bowel wall enhancement, mesenteric fluid, target configuration of bowel wall, serrated beak sign and smooth beak sign. Most of the studies included in this review did not discuss all these signs, which may be because of absence of CT signs or due to interpretive problems of 
Jha et al. Sensitivity and Specificity of CT and Its signs for Diagnosis of Strangulation in Patients with Acute Small Bowel...

the readers.

Our analysis revealed that mesenteric fluid, ascites, mesenteric haziness, bowel wall thickening, engorgement of mesenteric vessels and lack of bowel wall enhancement are the more sensitive CT signs in diagnosing strangulation in patients of acute SBO with a pooled sensitivity of $0.743,0.642,0.492,0.466$, 0.455 , and 0.404 respectively. Similarly, the presence of CT signs, lack of bowel wall enhancement, bowel wall thickening, mesenteric haziness, mesenteric fluid, engorgement of mesenteric vessels and the presence of ascites are the more specific CT finding in case of strangulation with a pooled specificity of 0.991 , $0.822,0.787,0.786,0.723$, and 0.702 respectively. While the CT signs like target configuration of bowel wall, serrated beak sign and smooth beak sign did not showed significant sensitivity in the diagnosis of strangulation with a pooled sensitivity of $0.308,0.395$, and 0.183 respectively, although their presence showed to be more specific in patients with strangulation in the setting of acute SBO with pooled specificity of 0.852 , 0.987 , and 0.380 respectively. The management of SBO is challenging because clinical, laboratory and plain radiographic evaluations cannot reliably establish or exclude the diagnosis of bowel ischemia, ${ }^{45,46}$ no preoperative clinical parameter proved to be highly sensitive, specific, or predictive of ischemia. Moreover, senior surgeon's clinical judgment correctly predicted ischemia in only $48 \%$ of the patients. ${ }^{1}$ Others have suggested that a high preoperative levels of interlukin- 6 are associated with intestinal ischemia, but the use of this laboratory test is not widespread. ${ }^{47,48}$ CT scan has been proposed to be more reliable preoperative diagnostic tool to detect ischemia in the presence of acute SBO. ${ }^{15,16,19,20,23}$ In this study, we found that CT scan is more sensitive and specific to diagnose ischemia in patients with acute SBO which is consistent with the previous studies. The role of individual CT signs of strangulation are well established by the previous studies but some studies has shown that they can also be present in cases of simple obstruction. ${ }^{15,24}$ Some authors suggest that a combination of signs may increase the diagnostic accuracy, ${ }^{15,16}$ and an aggregate scoring system may be attractive. This review also suggest that, although the previously mentioned signs of ischemia increases the sensitivity and specificity of CT in diagnosing ischemia individually, the effect on sensitivity and specificity of combination of two or more CT signs still needs to be proven.

This systemic review has several limitations. The included studies had differing inclusion and exclusion criteria (and therefore differing severity of illness). The difference of severity may present with more pathophysiological changes of bowel. Studies included in our analysis also differ with CT imaging technique, use of contrast media, radiological criteria used to diagnose ischemia or strangulation and blinding of the radiologists to the clinical data, differences among the radiologists regarding the $C T$ signs of strangulation, and the time of follow up for those patients who did not undergo surgery. An additional limitation is the small number of patients that were included in the analysis $(n=851)$, with largest number of patients 144, and smallest number being 19. The overall small sample size led to wide confidence intervals. Furthermore, it is possible that studies with positive effect tend to be published than those that do not, leading to publication bias. ${ }^{49} \mathrm{~A}$ verification bias may have also occurred because in most studies, high-risk patients results were evaluated. ${ }^{50}$ Several weakly positive studies may seem to add up to a strong positive result. The limitations of the individual included studies restrict the strength of the conclusion that can be drawn from this review. Given the variabilities in these studies and because none included prospective, randomized data, we believed that meta-analytic or higher level statistical comparisons were inappropriate and have simply provided pooled data. Although all studies used operative findings as their standard of reference for ischemia, the timing between CT scanning and surgery varied between studies and within studies themselves. This issue may have influenced the results of the studies as SBO findings during the course of illness can change over time. These variations may have contributed to the wide range of reported performance of CT. Furthermore, few studies failed to mention the presence or absence of the CT signs in false positive patients, as well as there are several outliers in the data presented in the included studies which need to be either discarded or reevaluated. In our review, we found that the strength of evidence provided by prospective studies are poorer than that of retrospective studies, which may be due to: (1) lesser number of prospective studies available for evaluation of CT scan, (2) the readers may missed important findings due to rush of time or lack of experience, and (3) the patients included in most of the retrospective studies are already diagnosed to have signs of bowel ischemia.

We could not discuss other issues like study cost, utilization of sources, optimal timing of CT scanning, effect of CT on the clinical outcome of patients in our analysis, and should be balanced against the accuracy of CT scanning in the relatively uncommon entity of bowel ischemia. Currently published articles do not address disparities in test performance between populations, and also there is very little number of articles discussing CT as well as all different CT signs together. Even though this review supports the reports of previous studies, due to heterogeneity of the CT scanning protocols, 
Jha et al. Sensitivity and Specificity of CT and Its signs for Diagnosis of Strangulation in Patients with Acute Small Bowel...

and heterogeneity of study populations, definitive recommendations must be limited. Development of clinical protocol for CT and different CT signs would require prospective studies of sufficient size that recruit patients of small bowel obstruction undergoing preoperative evaluation for the presence or absence of strangulation, stratify according to the results of CT and incorporate cost-effective analyses.

\section{CONCLUSIONS}

This review demonstrates that the sensitivity and specificity of CT is high in preoperative diagnosis of clinically suspected strangulation in the setting of acute SBO which are in accordance with the published studies. The sensitivity and specificity of CT and CT signs of ischemia are especially good for detecting strangulation in clinically suspected cases of acute SBO. Our analysis shows that "presence of mesenteric fluid" is the more sensitive, and "lack of bowel wall enhancement" is the more specific CT sign of strangulation. The metaanalysis of the literatures we conducted justifies large scale studies to validate the results obtained as well as to determine a clinical protocol.

\section{REFERENCES}

1. Mallo RD, Salem L, Lalani T, Flum DR. Computed tomography diagnosis of ischemia and complete obstruction in small bowel obstruction: a systematic review. J Gastrointest Surg. 2005;9(5):690-4

2. Bizer LS, Liebling RW, delany HM, Gliedman ML. Small bowel obstruction: the role of nonoperative treatment in simple intestinal obstruction and predictive criteria for strangulation obstruction. Surgery. 1981;89(4):407-13.

3. Sarr MG, Bulkley GB, Zuidema Gd. Preoperative recognition of intestinal obstruction: prospective evaluation of diagnostic capability. Am J Surg. 1983;145(1):176-82.

4. Playforth RH, Holloway JB, Griffen WO Jr. Mechanical small bowel obstruction: a plea for earlier surgical intervention. Ann Surg. 1970;171(5):783-8.

5. Wolfson PJ, Bauer JJ, Gelernt IM, Kreel I, Aufses AH Jr. Use of the long tube in the management of patients with small intestinal obstruction due to adhesions. Arch Surg 1985;120(9):1001-6.

6. Brolin RE, Krasna MJ, Mast BA. Use of tubes and radiographs in the management of small bowel obstruction. Ann Surg. 1987;206(2):126-33.

7. Megibow AJ. Bowel obstruction: evaluation with CT. Radiol Clin North Am. 1994;32(5):861-70.

8. Chakrabarty $\mathrm{P}$, tripathy $\mathrm{B}$, Panda $\mathrm{K}$. Acute intestinal obstruction (a review of 1020 operated cases). J Indian Med Assoc. 1976;67(3):64-9.

9. Laws HL, Aldrete JS. Small bowel obstruction: a review of 465 cases. South Med J. 1976;69(6):733-4.

10. Bogusevicius A, grinkevicius A, Maleckas A, Pundzius J. The role of D-dimer in the diagnosis of strangulated small bowel obstruction. Medicina (kaunas). 2007;43(11):850-4.

11. Silen W, Hein M, Goldman L. Strangulation obstruction of the small intestine. Arch Surg 1962;85:121-9.

12. Leffall LD, Syphax B. Clinical aids in strangulation intestinal obstruction. Am J Surg, 1970;120(6):756-9.

13. Shatila AH, Chamberlain BE, Webb WR. Current status of diagnosis and management of strangulation obstruction of small bowel. Am J Surg. 1976;132(3):299-303.

14. Kim JH, Ha HK, Kim JK, Eun HW, Park KB, Kim BS, et al. Usefulness of known computed tomography and clinical criteria for diagnosing strangulation in small-bowel obstruction: analysis of true and false interpretation groups in computed tomography. World J Surg. 2004; 8(1):63-8.

15. Ha HK, Kim JS, Lee MS, Lee HJ, Jeong YK, Kim PN, et al. Differentiation of simple and strangulated small-bowel obstructions: usefulness of known CT criteria. Radiology. 1997;204(2):507-12.

16. Donckier V, Closset J, Van Gansbeke D, Zalcman M, Sy M, Houben JJ, Lambilliotte JP. Contribution of computed tomography to decision making in the management of adhesive small bowel obstruction. BrJSurg. 1998;85(8):1071-4.

17. Zalcman M, Sy M, Donckier V, Closset J, Gansbeke DV. Helical CT signs in the diagnosis of intestinal ischemia in small-bowel obstruction. AJR Am J Roentgenol. 2000;175(6):1601-7.

18. Sheedy SP, Earnest F $4^{\text {th }}$ Fletcher JG, Fidler JL, Hoskin TL. CT of small-bowel ischemia associated with obstruction in emergency department patients: diagnostic performance evaluation. Radiology. 2006;241(3):729-36.

19. Balthazar EJ, Liebeskind ME, Macari M. Intestinal ischaemia in patients in whom small bowel obstruction is suspected: evaluation of accuracy, limitations and clinical implications of CT in diagnosis. Radiology. 1997;205(2): 519-22.

20. Balthazar EJ, Birnbaum BA, Megibow AJ, Gordon RB, Whelan CA, Hulnick DH. Closed-loop and strangulating intestinal obstruction: CT signs. Radiology. 1992;185(3):769-75.

21. Balthazar EJ. CT of small-bowel obstruction. AJR Am J Roentgenol. 1994;162(2):255-61.

22. Taourel PG, Deneuville M, Pradel JA, Regent D, Bruel JM. Acute mesenteric ischemia: diagnosis with contrast-enhanced CT. Radiology. 1996;199(3):632-6.

23. Frager D, Baer JW, Medwid SW, Rothpearl A, Bossart $P$. Detection of intestinal ischemia in patients with acute small-bowel obstruction due to adhesions or hernia: efficacy of CT. AJR Am J Roentgenol. 1996;166(1):67-71. 
24. Ha HK, Park CH, Kim SK, Chun CS, Kim IC, Lee HK, et al. CT analysis of intestinal obstruction due to adhesions: early detection of strangulation. J Comput Assist Tomogr. 1993;17(3):386-9.

25. Zalcman M, Van Gansbeke D, Lalmand B, Braudé P, Closset J, Struyven J. Delayed enhancement of the bowel wall: a new CT sign of small bowel strangulation. J Comput Assist Tomogr. 1996;20(3):379-81.

26. Alpern MB, Glazer GM, Francis IR. Ischemic or infarcted bowel: CT findings. Radiology. 1988;166(1 pt 1):149-52.

27. Fisher JK. Computed tomographic diagnosis of volvulus in intestinal malrotation. Radiology. 1981;140(1):145-6.

28. Jaramillo D, Raval B. CT diagnosis of primary small bowel volvulus. AJR Am J Roentgenol. 1986; 147(5): 941-2.

29. Jaeschke R, Guyatt G, Sackett DL. Users' guides to the medical literature. III. How to use an article about a diagnostic test. A. Are the results of the study valid? Evidence-Based Medicine Working Group. JAMA. 1994;271(5):389-91.

30. Jaeschke R, Guyatt G, Sackett DL. Users' guides to the medical literature. III. How to use an article about a diagnostic test. B. What are the results and will they help me in caring for my patients? The Evidence-Based Medicine Working Group. JAMA. 1994;271(9):703-7.

31. Whiting P, Rutjes AW, Reitsma JB, Bossuyt PM, Kleijnen J. The development of QUADAS: a tool for the quality assessment of studies of diagnostic accuracy included in systematic reviews. BMC Med Res Methodol. 2003;3:25.

32. Yen $\mathrm{CH}$, Chen JD, Tui CM, Chou YH, Lee CH, Chang CY,et al. Internal hernia: computed tomography diagnosis and differentiation from adhesive small bowel obstruction. JChin Med Assoc. 2005;68(1):21-8.

33. Catel L, Lefevre F, Lauren V, canard L, Bresler L, Guillemin $\mathrm{F}$, et al. Small bowel obstruction from adhesions: which CT severity criteria to research? J Radiol. 2003;84(1):27-31.

34. Taourel PG, Fabre JM, Pradel JA, Seneterre EJ, Megibow AJ, Bruel JM. Value of CT in the diagnosis and management of patients with suspected acute small bowel obstruction. AJR Am J Roentgenol. 1995;165(5):1187-92.

35. Evers BM. Small bowel. In: Townsend Cea, ed. Sabiston Textbook of Surgery. 16th ed. Philadelphia: W.B. Saunders Company; 2001:pp 883-88.

36. Bass KN, Jones B, Bulkley GB. Current management of small-bowel obstruction. Adv Surg. 1997;31:1-34.

37. Fevang BT, Jensen D, Svanes K, Viste A. Early operation or conservative management of patients with small bowel obstruction? Eur J Surg 2002;168(8-9):475-81.
38. Frager D, Medwid SW, Baer JW, Mollinelli B, Friedman M. CT of small-bowel obstruction: value in establishing the diagnosis and determining the degree and cause. AJR Am J Roentgenol. 1994;162(1):37-41.

39. Fukuya T, Hawes DR, Lu CC, Chang PJ, Barloon TJ. CT diagnosis of small bowel obstruction: efficacy in 60 patients. AJR Am J Roentgenol. 1992;158(4):765-9.

40. Fevang BT, Fevang J, Stangeland L, Soreide O, Svanes K, Viste A. Complications and death after surgical treatment of small bowel obstruction: a 35-year institutional experience. Ann Surg. 2000;231(4):529-37.

41. Makita O, Ikushima I, Matsumoto N, Arikawa K, Yamashita Y, Takahashi M. CT differentiation between necrotic and nonnecrotic small bowel in closed loop and strangulating obstruction. Abdom Imaging. 1999;24(2):120-4.

42. Wiesner W, Khhurana B, Ji H, Ros PR. CT of acute bowel ischemia. Radiology. 2003;226(3):635-50.

43. Megibow AJ, Balthazar EJ, Cho KC, Medwid SW, Birnbaum BA, Noz ME. Bowel obstruction: evaluation with CT. Radiology. 1991;180(2):313-8.

44. Rubesin SE, Herlinger H. CT evaluation of bowel obstruction: a landmark article - implication for the future. Radiology. 1991;180(2):307-8.

45. Cheadle WG, Garr EE, Richardson JD. The importance of early diagnosis of small bowel obstruction. Am Surg. 1988;54(9):565-9.

46. Gough IR. Strangulating adhesive small bowel obstruction with normal radiographs. Br J Surg. 1978;65(6):431-4.

47. Sutherland F, Cunningham H, Pontikes L, Parsons L, Klassen J. Elevated serum interleukin 6 levels in patients with acute intestinal ischemia. Hepatogastroenterology. 2003;50(50):419-21.

48. Firoozmand E, Fairman N, Sklar J, Waxman K. Intravenous interleukin-6 levels predict need for laparotomy in patients with bowel obstruction. Am Surg. 2001;67(12):1145-9.

49. Irwig L, Macaskill P, Glasziou P, Fahey M. Meta-analytic methods for diagnostic test accuracy. J Clin Epidemiol. 1995;48(1):119-30.

50. Begg $\mathrm{CB}$, McNeil BJ. Assessment of radiologic tests: control of bias and other design considerations. Radiology. 1988;167(2):565-9. 